\title{
Determination of sinus pericranii resectability by external compression during angiography: technical note
}

\author{
Jason A. Ellis, MD, Juan C. Mejia Munne, BS, Neil A. Feldstein, MD, and Philip M. Meyers, MD \\ Department of Neurological Surgery, Columbia University Medical Center, New York
}

Sinus pericranii is an uncommon congenital cranial venous malformation that may become symptomatic in the pediatric population. Both dominant and accessory sinus pericranii, as determined by the intracranial venous drainage pattern, have been described. The dominant variety drain a significant proportion of the intracranial venous outflow while the accessory variety have minimal or no role in this. Classic teachings hold that dominant sinus pericranii should never be treated while accessory sinus pericranii may be safely obliterated. This determination of dominance is solely based on a qualitative assessment of standard venous phase catheter cerebral angiography, leaving some doubt regarding the actual safety of obliteration. In this paper the authors describe a simple and unique method for determining whether intracranial venous outflow may be compromised by sinus pericranii treatment. This involves performing catheter angiography while the lesion is temporarily obliterated by external compression. Analysis of intracranial venous outflow in this setting allows visualization of angiographic changes that will occur once the sinus pericranii is permanently obliterated. Thus, the safety of surgical intervention can be more fully appraised using this technique.

http://thejns.org/doi/abs/10.3171/2015.6.PEDS15183

KEY WORDS angiography; congenital; embolization; surgery; venous malformation; vascular disorders

$\mathrm{S}$ INUS pericranii is a vascular malformation composed of extracranial veins and a prominent venous varix connected to an intracranial venous sinus via emissary veins. Although rare traumatic associations have been reported, most suggest a congenital etiology for this malformation, given its association with developmental venous anomalies and other vascular lesions. ${ }^{22,23,41,45} \mathrm{Si}$ nus pericranii was first described by Hecker in 1845 and elaborated by Stromeyer in 1850 . Of this curiosity Stromeyer wrote that it "...consists of a blood bag on the skull, which stands in connection with the veins of the diploe and through these with the sinuses of the brain...." ${ }^{2,57,58}$

Due to the rarity of sinus pericranii, its pathogenesis and natural history remain unclear. Thus, the optimal management strategy for this lesion is also unknown. Symptomatic sinus pericranii or those resulting in a cosmetic deformity are strongly considered for surgical obliteration. Reports of an association with hemorrhage, sinus thrombosis, intracranial hypertension, and infection have led some to aggressively treat asymptomatic sinus pericranii. ${ }^{17,45}$ Decision making is complicated by difficulty in determining which sinus pericranii are safe for surgical intervention.

The angioarchitectural classification of sinus pericranii into dominant or accessory varieties is an attempt to facilitate making a determination about which lesions may be safely obliterated. The late Pierre Lasjaunias and coworkers defined dominant lesions as those where the main stream of contrast material flow uses the sinus pericranii to drain the brain, bypassing the usual venous outlets. In contrast, accessory lesions were defined as those in which only a small part of the venous outflow occurs through the extradiploic vessels. ${ }^{23}$ This qualitative angiographic designation has become the main factor in determining whether a sinus pericranii can be treated. It is believed that dominant sinus pericranii must be preserved while accessory sinus pericranii are candidates for surgical intervention. ${ }^{45}$

Although we have found the aforementioned classification scheme helpful for surgical decision making, we have developed a protocol for additional angiographic interrogation of sinus pericranii that may further ensure the safety of intervention. The lesion compression technique we describe here enables one to make an assessment of the adequacy of intracranial venous outflow during temporary functional absence of a sinus pericranii. This additional assessment allows the surgeon or interventional specialist to proceed confidently with obliteration of a sinus pericranii deemed redundant within the intracranial circulation. 

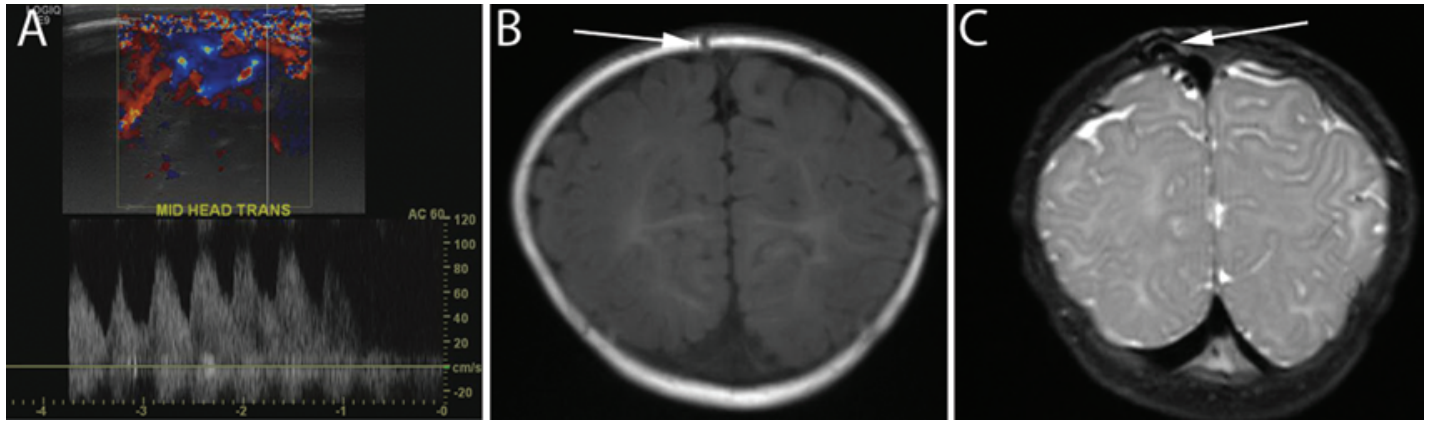

FIG. 1. Noninvasive imaging of sinus pericranii. Doppler ultrasonography of the right parietal mass demonstrated marked vascularity within the scalp and communication with the superior sagittal sinus (A). Coronal T1- (B) and T2-weighted (C) brain MRI confirmed the presence of an epicranial venous varix providing communication (arrow) between scalp veins and the superior sagittal sinus. Figure is available in color online only.

\section{Presentation and Noninvasive Imaging}

A 7 month-old, healthy female infant presented electively with a pulsatile, right parasagittal scalp mass (Video 1).

VIDEO 1. Clip showing pulsatile scalp mass. Copyright Jason A.

Ellis. Published with permission. Click here to view.

As per the parents' assessment, modest growth over several months was noted. Doppler ultrasonography demonstrated spectral waveforms within the scalp concerning for a vascular malformation (Fig. 1A). Subsequent MRI showed a small tangle of prominent flow voids in the region of interest, with 1 vessel traversing the parietal calvaria and draining into the superior sagittal sinus (Fig. 1B and C).

\section{Angiographic Technique}

Although a diagnosis of sinus pericranii was made noninvasively and the typically benign nature of the condition was described, the parents wished to proceed with intervention if deemed safe. Thus, catheter cerebral angiography was performed to more fully assess the angioarchitecture of the sinus pericranii and the associated venous drainage pattern of the brain.

Given the patient's young age, the procedure was performed under general endotracheal anesthesia to ensure high-resolution imaging without motion artifacts and to allow for direct transfer to the operating suite for excision if deemed appropriate. Following sterile preparation and draping, a 4-Fr arterial sheath was placed in the right common femoral artery using a modified Seldinger technique with a micropuncture needle. A 4-Fr hockey-stick catheter was passed over a Bentson guidewire to sequentially obtain standard 6-vessel arteriography, including the bilateral common and internal carotid arteries as well as the bilateral vertebral arteries. The sinus pericranii was noted to opacify at the expected interval in the venous phase without arteriovenous shunting. This occurred in conjunction and connection with the superior sagittal sinus draining to the scalp veins (Fig. 2A and B). The sinus pericranii drained only a small portion of the intracranial venous outflow and was, by definition, accessory. ${ }^{23}$

A pressure dressing was subsequently applied to the scalp directly above the sinus pericranii. Repeat arteriography with external compression in place demonstrated normal arteriovenous transit opacification of the intracranial vessels, but no opacification of the extracranial scalp vessels through the sinus pericranii (Fig. $2 \mathrm{C}$ and D). The intracranial venous drainage pattern, including that of the superior sagittal sinus, was normal, suggesting that the sinus pericranii may be safely excised.

\section{Surgical Excision}

The patient was transported to the operating theater while under the same general anesthesia used to perform catheter angiography. Positioning was prone with the head
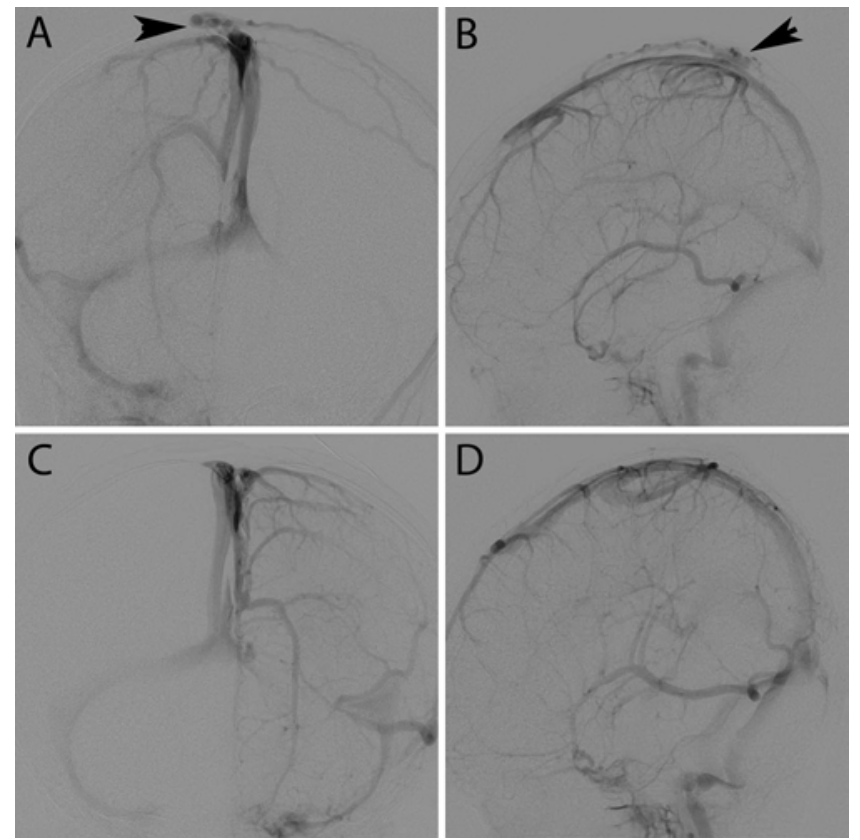

FIG. 2. Angiographic assessment of sinus pericranii involvement with intracranial venous outflow. Anteroposterior (A) and lateral (B) late venous phase angiograms show the sinus pericranii (arrowhead) and associated dilated scalp veins. After external compression of the sinus pericranii was applied, repeat angiography (C and D) showed a normal pattern of intracranial venous outflow. 
on a cerebellar headrest (Fig. 3). After usual prepping and draping, a slightly parasagittal incision was made near the vertex slightly to the right of midline with care taken not to incise the underlying venous anomaly. Using bipolar and sharp dissection, numerous scalp veins as well as a single feeder to the sinus pericranii from the sagittal sinus was identified, cauterized, and ligated. The subgaleal plane was swept circumferentially down to the level of the bone, with stripping of the periosteum a distance of 3-4 $\mathrm{cm}$ radially. The pericranium was reduced with cautery to the edge of the skull defect through which the sagittal sinus communicated. At this point, the pulsations in the scalp from the malformation were completely absent. Final inspection, putting the patient in the Trendelenburg position, and executing a Valsalva maneuver indicated no evidence of residual sinus pericranii or abnormal scalp feeders/tributaries. The patient tolerated the procedure well and was discharged home neurologically intact on the first postoperative day.

\section{Discussion}

Although more than 100 cases of sinus pericranii have been reported in the literature, its relative rarity has precluded standardization of diagnosis, classification, and management. ${ }^{1-19,21-56,59,60,62-64}$ Not surprisingly, the majority of reported cases are unclassified with respect to dominance. Most reports suggest that sinus pericranii are clinically benign lesions that predominantly raise cosmetic concerns. However, rare reports of hemorrhage (spontaneous and traumatic), infection, air embolism, intracranial hypertension, and sinus thrombosis have led some practitioners and patients to pursue aggressive intervention. Accurate clinical and radiographic assessment of sinus pericranii is therefore essential prior to intervening on this probably benign lesion.

Févre and Modec proposed the first classification system for sinus pericranii in $1936 .{ }^{20}$ Their scheme included descriptions of sinus pericranii as: 1) closed systems arising from and draining into intracranial sinuses; 2) drainer systems that act as collaterals for intracranial flow; and 3) extracranial lesions draining into intracranial sinuses. Gandolfo and colleagues proposed a more clinically useful categorization, explaining that dominant sinus pericranii are untreatable because they serve as a major venous outflow channel to the intracranial compartment, whereas accessory sinus pericranii are highly treatable because only a small portion of the intracranial venous outflow traverses them. ${ }^{23}$ Classification of sinus pericranii as dominant or accessory has arguably been the most important criterion for determining whether this anomaly may be safely obliterated..$^{23,45}$ In our opinion, the compression technique we describe for functionally, but reversibly, obliterating sinus pericranii provides additional assurance that intracranial venous outflow will not be compromised by resection or embolization. Discordant pre- and postcompression venous phase angiography favors conservative management. Although not performed in our case, use of awake neurological testing and/or evoked potential monitoring may provide additional assurances.

It is notable that various compression maneuvers in pa-

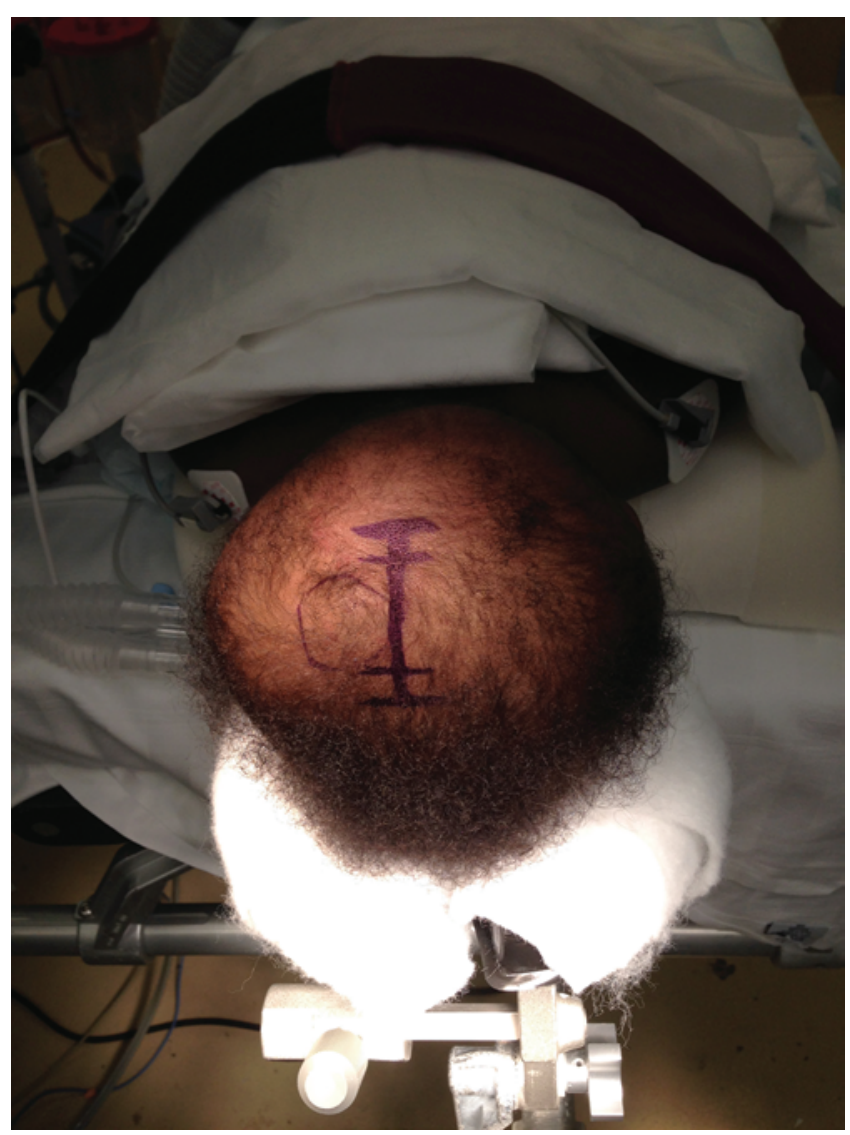

FIG. 3. Patient position for resection. The patient was positioned prone with the head on a cerebellar headrest. The lesion was outlined and the planned linear parasagittal incision was marked as shown. Figure is available in color online only.

tients with sinus pericranii have been previously described. However, we are unaware of these techniques being used in the manner we describe to determine therapeutic options. Volkman in 1950 used direct compression to differentiate true sinus pericranii from pseudo-sinus pericranii (hemangioma, cavernoma, etc.). ${ }^{61}$ Kaido et al. described compression of the jugular veins during cerebral angiography, but this maneuver provided little information for clinical decision making. ${ }^{27}$ Madsen and colleagues employed compression of a cutis aplasia lesion associated with a sinus pericranii during CT venography to assess intracranial drainage patterns. ${ }^{38}$

\section{Conclusions}

Sinus pericranii are benign congenital vascular malformations associated with a rare incidence of complications. Treatment via endovascular or open surgical methods should be attempted only after angiographic confirmation that the lesion is accessory. We believe that direct sinus pericranii compression during catheter angiography is a safe way to determine if intracranial venous outflow may be normal after lesion treatment. Validation of this technique in additional patients will be necessary to confirm this finding. 


\section{References}

1. Aburto-Murrieta Y, Bonifacio-Delgadillo D, Balderrama Bañares J, Zenteno Castellanos MA: Sinus pericranii: case report. Vasc Endovascular Surg 45:103-105, 2011

2. Akram H, Prezerakos G, Haliasos N, O’Donovan D, Low H: Sinus pericranii: an overview and literature review of a rare cranial venous anomaly (a review of the existing literature with case examples). Neurosurg Rev 35:15-26, 2012

3. Anegawa S, Hayashi T, Torigoe R, Nakagawa S, Ogasawara T: Sinus pericranii with severe symptom due to transient disorder of venous return-case report. Neurol Med Chir (Tokyo) 31:287-289, 1991

4. Arrues MA, Dickmann GH, Pataro VF: Sinus percranii (five cases). Angiology 7:186-193, 1956

5. Beers GJ, Carter AP, Ordia JI, Shapiro M: Sinus pericranii with dural venous lakes. AJNR Am J Neuroradiol 5:629_ 631, 1984

6. Bhutada S, Lokeshwar MR, Pandey A, Kulkarni M: Sinus pericranii: a case report and review of literature. Indian $\mathbf{J}$ Pediatr 79:1523-1525, 2012

7. Bigot JL, Iacona C, Lepreux A, Dhellemmes P, Motte J, Gomes H: Sinus pericranii: advantages of MR imaging. Pediatr Radiol 30:710-712, 2000

8. Bollar A, Allut AG, Prieto A, Gelabert M, Becerra E: Sinus pericranii: radiological and etiopathological considerations. Case report. J Neurosurg 77:469-472, 1992

9. Bonioli E, Bellini C, Palmieri A, Fondelli MP, Tortori Donati P: Radiological case of the month. Sinus pericranii. Arch Pediatr Adolesc Med 148:607-608, 1994

10. Brisman JL, Niimi Y, Berenstein A: Sinus pericranii involving the torcular sinus in a patient with Hunter's syndrome and trigonocephaly: case report and review of the literature. Neurosurgery 55:433, 2004

11. Brook AL, Gold MM, Farinhas JM, Goodrich JT, Bello JA: Endovascular transvenous embolization of sinus pericranii. Case report. J Neurosurg Pediatr 3:220-224, 2009

12. Buxton N, Vloeberghs M: Sinus pericranii. Report of a case and review of the literature. Pediatr Neurosurg 30:96-99, 1999

13. Carpenter JS, Rosen CL, Bailes JE, Gailloud P: Sinus pericranii: clinical and imaging findings in two cases of spontaneous partial thrombosis. AJNR Am J Neuroradiol 25:121-125, 2004

14. Cheraghi N, Delano S, Csikesz C, Dundamadappa S, Wiss K: Sinus pericranii with a hair collar sign. Pediatr Dermatol 31:397-398, 2014

15. Chowdhury FH, Haque MR, Kawsar KA, Sarker MH, Momtazul Haque AF: Surgical management of scalp arteriovenous malformation and scalp venous malformation: An experience of eleven cases. Indian J Plast Surg 46:98-107, 2013

16. Curnes JT: Sinus pericranii: demonstration using threedimensional surface shading. J Comput Assist Tomogr 26:285-286, 2002

17. David LR, Argenta LC, Venes J, Wilson J, Glazier S: Sinus pericranii. J Craniofac Surg 9:3-10, 1998

18. Desai K, Bhayani R, Goel A, Muzumdar D: Sinus pericranii in the frontal region: a case report. Neurol India 49:305307, 2001

19. Drosou A, Benjamin L, Linfante I, Mallin K, Trowers A, Wakhloo AK, et al: Infantile midline facial hemangioma with agenesis of the corpus callosum and sinus pericranii: another face of the PHACE syndrome. J Am Acad Dermatol 54:348-352, 2006

20. Févre M, Modec L: Sinus pericranii et tumeurs vasculaires extracraniennes communiquant avec la circulation intracranienne. J Chir (Paris) 47:561-588, 1936

21. Frassanito P, Massimi L, Tamburrini G, Caldarelli M, Pedi- celli A, Di Rocco C: Occipital sinus pericranii superseding both jugular veins: description of two rare pediatric cases. Neurosurgery 72:E1054-E1058, 2013

22. Gabikian P, Clatterbuck RE, Gailloud P, Rigamonti D: Developmental venous anomalies and sinus pericranii in the blue rubber-bleb nevus syndrome. Case report. J Neurosurg 99:409-411, 2003

23. Gandolfo C, Krings T, Alvarez H, Ozanne A, Schaaf M, Baccin $\mathrm{CE}$, et al: Sinus pericranii: diagnostic and therapeutic considerations in 15 patients. Neuroradiology 49:505-514, 2007

24. Gezina Sas AM, van Kooten F: Teaching NeuroImages: sinus pericranii. Neurology 72:e66, 2009

25. Hsu SW, Chaloupka JC: Atretic parietal cephalocele associated with sinus pericranii: embryological consideration. Brain Dev 34:325-328, 2012

26. Jung S, Lee JK, Kim SH, Kim JH, Kang SS, Lee JH: Parietal sinus pericranii: case report and technical note. Surg Neurol 54:270-273, 2000

27. Kaido T, Kim YK, Ueda K: Diagnostic and therapeutic considerations for sinus pericranii. J Clin Neurosci 13:788-792, 2006

28. Kamble RB, Venkataramana NK, Naik L, Shailesh, Shetty R: Sinus pericranii presenting with macrocephaly and mental retardation. J Pediatr Neurosci 5:39-41, 2010

29. Kanavaki A, Dhouib A, Zand T, Anooshiravani M, Hanquinet S: Sinus pericranii: a scalp mass in a 6-month-old boy. Pediatr Neurosurg 48:126-128, 2012

30. Kessler IM, Esmanhoto B, Riva R, Mounayer C: Endovascular transvenous embolization combined with direct punction of the sinus pericranii. A case report. Interv Neuroradiol 15:429-434, 2009

31. Kim YJ, Kim IO, Cheon JE, Lim YJ, Kim WS, Yeon KM: Sonographic features of sinus pericranii in 4 pediatric patients. J Ultrasound Med 30:411-417, 2011

32. Kimiwada T, Hayashi T, Sanada T, Shirane R, Tominaga T: Surgical treatment of scaphocephaly with sinus pericranii. Neurol Med Chir (Tokyo) 53:121-125, 2013

33. Kurosu A, Wachi A, Bando K, Kumami K, Naito S, Sato K: Craniosynostosis in the presence of a sinus pericranii: case report. Neurosurgery 34:1090-1093, 1994

34. Little FM, Segall HD, McComb JG: Sinus pericranii discovered at surgery for anticipated epidermoid cyst of the skull: a case report. J Child Neurol 2:71-72, 1987

35. Lo PA, Besser M, Lam AH: Sinus pericranii: a clinical and radiological review of an unusual condition. J Clin Neurosci 4:247-252, 1997

36. Luker GD, Siegel MJ: Sinus pericranii: sonographic findings. AJR Am J Roentgenol 165:175-176, 1995

37. Macit B, Burrows PE, Yilmaz S, Orbach DB, Mulliken JB, Alomari AI: Cerebrofacial venous anomalies, sinus pericranii, ocular abnormalities and developmental delay. Interv Neuroradiol 18:153-157, 2012

38. Madsen JR, Robertson RL, Bartlett R: Surgical management of cutis aplasia with high-flow sinus pericranii. Pediatr Neurosurg 28:79-83, 1998

39. Mahindu AJ, Guazzo EG: Blocked ventriculoperitoneal shunt causing raised intracranial pressure diagnosed by prominent sinus pericranii. J Clin Neurosci 16:1686-1687, 2009

40. Marras C, McEvoy AW, Grieve JP, Jäger HR, Kitchen ND, Villani RM: Giant temporo-occipital sinus pericranii. A case report. J Neurosurg Sci 45:103-109, 2001

41. Mitsukawa N, Satoh K, Hayashi T, Furukawa Y, Suse T, Uemura T, et al: Sinus pericranii associated with craniosynostosis. J Craniofac Surg 18:78-84, 2007

42. Nomura S, Kato S, Ishihara H, Yoneda H, Ideguchi M, Suzuki M: Association of intra- and extradural developmental venous anomalies, so-called venous angioma and sinus pericranii. Childs Nerv Syst 22:428-431, 2006 
43. Ohta $T$, Waga $S$, Handa $H$, Nishimura S, Mitani T: Sinus pericranii. J Neurosurg 42:704-712, 1975

44. Park SC, Kim SK, Cho BK, Kim HJ, Kim JE, Phi JH, et al: Sinus pericranii in children: report of 16 patients and preoperative evaluation of surgical risk. J Neurosurg Pediatr 4:536-542, 2009

45. Pavanello M, Melloni I, Antichi E, Severino M, Ravegnani M, Piatelli G, et al: Sinus pericranii: diagnosis and management in 21 pediatric patients. J Neurosurg Pediatr 15:6070, 2015

46. Raheja A, Satyarthee GD, Sharma BS: Single, small, spontaneous, accessory, closed type, frontal sinus pericranii in a child: favorable outcome with surgical excision. Neurol India 61:680-683, 2013

47. Rangel-Castilla L, Krishna C, Klucznik R, Diaz O: Endovascular embolization with Onyx in the management of sinus pericranii: a case report. Neurosurg Focus 27(5):E13, 2009

48. Rizvi M, Behari S, Singh RK, Gupta D, Jaiswal AK, Jain $\mathrm{M}$, et al: Sinus pericranii with unusual features: multiplicity, associated dural venous lakes and venous anomaly, and a lateral location. Acta Neurochir (Wien) 152:2197-2204, 2010

49. Rozen WM, Joseph S, Lo PA: Spontaneous involution of two sinus pericranii - a unique case and review of the literature. $\mathbf{J}$ Clin Neurosci 15:833-835, 2008

50. Saba R, Senussi MH, Alwakkaf A, Brown H: Sinus pericranii in a young adult with chronic headache. BMJ Case Rep 2013:bcr2013010165, 2013

51. Sadler LR, Tarr RW, Jungreis CA, Sekhar L: Sinus pericranii: CT and MR findings. J Comput Assist Tomogr 14:124127, 1990

52. Sakai K, Namba K, Meguro T, Mandai S, Gohda Y, Sakurai $\mathrm{M}$, et al: Sinus pericranii associated with a cerebellar venous angioma-case report. Neurol Med Chir (Tokyo) 37:464467, 1997

53. Schenk B, Brouwer PA: Bilateral frontal sinus pericranii with an intratabular course. A case report. Interv Neuroradiol 16:179-182, 2010

54. Shah AK, Camilleri AC, Kirkpatrick P, Javaid M: Sinus pericranii-“Don't judge a lump by its surface". J Craniofac Surg 21:1585-1586, 2010

55. Sheu M, Fauteux G, Chang H, Taylor W, Stopa E, RobinsonBostom L: Sinus pericranii: dermatologic considerations and literature review. J Am Acad Dermatol 46:934-941, 2002

56. Spektor S, Weinberger G, Constantini S, Gomori JM, BeniAdani L: Giant lateral sinus pericranii. Case report. J Neurosurg 88:145-147, 1998
57. Stromeyer L: About sinus pericranii (translating of original 1850 text). Surg Neurol 40:3-4, 1993

58. Stromeyer L: Ueber Sinus pericranii. Dtsch Klin 2:160, 1850

59. Sty JR, Murphy J, Thorp S: Brain scintigraphy: sinus pericranii. Clin Nucl Med 6:184-185, 1981

60. Vaquero J, de Sola RG, Martínez R: Lateral sinus pericranii. Case report. J Neurosurg 58:139-140, 1983

61. Volkmann J: Ein beitrag zur sogenannten sinus pericranii (Stromeyer). Zentralbl Chir 75:1389-1394, 1950

62. Wen CS, Chang YL, Wang HS, Kuo MF, Tu YK: Sinus pericranii: from gross and neuroimaging findings to different pathophysiological changes. Childs Nerv Syst 21:482-488, 2005

63. Yanik B, Keyik B, Conkbayir I, Kuru AA, Hekimodlu B: Sinus pericranii: color Doppler ultrasonographic findings. J Ultrasound Med 25:679-682, 2006

64. Zeikus P, Robinson-Bostom L, Stopa E: Primary cutaneous meningioma in association with a sinus pericranii. J Am Acad Dermatol 54 (2 Suppl):S49-S50, 2006

\section{Disclosure}

The authors report no conflict of interest concerning the materials or methods used in this study or the findings specified in this paper.

\section{Author Contributions}

Conception and design: Ellis. Acquisition of data: Ellis, Mejia Munne. Analysis and interpretation of data: Ellis, Feldstein, Meyers. Drafting the article: Ellis, Mejia Munne. Critically revising the article: Ellis, Feldstein, Meyers. Reviewed submitted version of manuscript: Ellis, Feldstein, Meyers. Approved the final version of the manuscript on behalf of all authors: Ellis.

\section{Supplemental Information Video}

Video 1. https://vimeo.com/140821075.

\section{Correspondence}

Jason A. Ellis, Department of Neurological Surgery, Columbia University Medical Center, Neurological Institute of New York, 710 W. 168th St., New York, NY 10032.email: jae2109@ columbia.edu. 